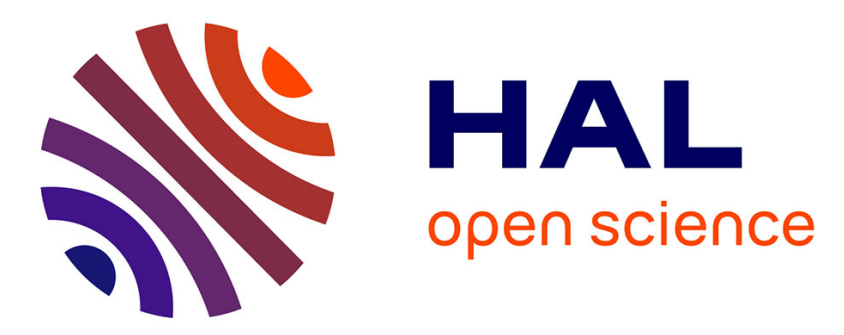

\title{
Utilisation du laser dans l'analyse isotopique d'un échantillon de DLi
}

\author{
P. Langer, B. Pin, G. Tonon
}

\section{To cite this version:}

P. Langer, B. Pin, G. Tonon. Utilisation du laser dans l'analyse isotopique d'un échantillon de DLi. Revue de Physique Appliquée, 1968, 3 (4), pp.405-413. 10.1051/rphysap:0196800304040500 . jpa00242879

\section{HAL Id: jpa-00242879 https://hal.science/jpa-00242879}

Submitted on 1 Jan 1968

HAL is a multi-disciplinary open access archive for the deposit and dissemination of scientific research documents, whether they are published or not. The documents may come from teaching and research institutions in France or abroad, or from public or private research centers.
L'archive ouverte pluridisciplinaire HAL, est destinée au dépôt et à la diffusion de documents scientifiques de niveau recherche, publiés ou non, émanant des établissements d'enseignement et de recherche français ou étrangers, des laboratoires publics ou privés. 


\title{
UTILISATION DU LASER \\ DANS L'ANALYSE ISOTOPIQUE D'UN ÉGHANTILLON DE DLi
}

\author{
Par P. LANGER, B. PIN et G. TONON, \\ Commissariat à l'Énergie Atomique. \\ (Reçu le 2 juillet 1968.)
}

\begin{abstract}
Résumé. - Les premiers résultats relatifs à une méthode d'analyse isotopique d'un échantillon de DLi sont exposés. Dans cette méthode, on utilise le faisceau d'un laser déclenché, focalisé sur la surface de la cible en deutérure de lithium. On effectue, à l'aide d'un analyseur électrostatique, la détection des ions $\mathrm{H}^{+}$et $\mathrm{D}^{+}$provenant du plasma créé. La mesure des intensités ioniques permet de connaître le rapport :
\end{abstract}

$$
\eta=\frac{\text { nombre d'atomes d'hydrogène }}{\text { nombre d'atomes de deutérium }}
$$

au point d'impact du faisceau laser.

Une étude de l'évolution de $\eta$ en fonction de la profondeur est rendue possible par l'usinage sous vide de la cible de deutérure de lithium.

Abstract. - Isotopic analysis of a DLi specimen has been performed using a Q-switched laser beam. Ion species are selected with an electrostatic analyser. The ratio :

$$
\eta=\frac{\text { number of hydrogen atoms }}{\text { number of deuterium atoms }}
$$

has been studied for a series of laser impacts as a function of the depth of the vacuum machined DLi target.

\section{INTRODUCTION}

Le faisceau d'un laser déclenché est focalisé à la surface de la cible en deutérure de lithium dont on veut faire l'analyse isotopique, c'est-à-dire, en ce qui nous concerne, déterminer le rapport :

$$
\eta=\frac{\text { nombre d'atomes d'hydrogène }}{\text { nombre d'atomes de deutérium }} \text {. }
$$

Des études antérieures relatives à l'interaction lasercible métallique [1] à [5] ont montré que l'on crée un plasma contenant les espèces ionisées de tous les atomes présents au point d'impact du faisceau laser. L'expansion du plasma est accompagnée d'une émission importante d'ions ayant acquis une certaine énergie cinétique. Ce mécanisme d'émission constitue une véritable source d'ions fonctionnant par impulsions.

Le rapport $\eta$ est déterminé en détectant les ions $\mathrm{H}^{+}$ et $\mathrm{D}^{+}$, qui se séparent à l'aide d'un analyseur électrostatique couplé à un dispositif à temps de vol.
On peut admettre que l'hydrogénation de la cible n'est pas homogène en profondeur, car elle se fait à travers la surface en contact avec le milieu extérieur. Ceci a été vérifié en mesurant la variation de $\eta$ en fonction de la profondeur de la cible par usinage de la surface. Notons que le laser permet d'effectuer une analyse ponctuelle à la surface de l'échantillon.

\section{DISPOSITIF EXPERIMENTAL}

Le dispositif expérimental est schématisé figure $1 \mathrm{a}$. Il comporte essentiellement deux parties :

1. La source ionique. - Nous la considérons constituée par le laser, son objectif de concentration et la cible à analyser.

a) Cible en deutérure de lithium. - La cible en deutérure de lithium est placée dans un double container représenté figure $1 \mathrm{~b}$. Une boîte étanche en 


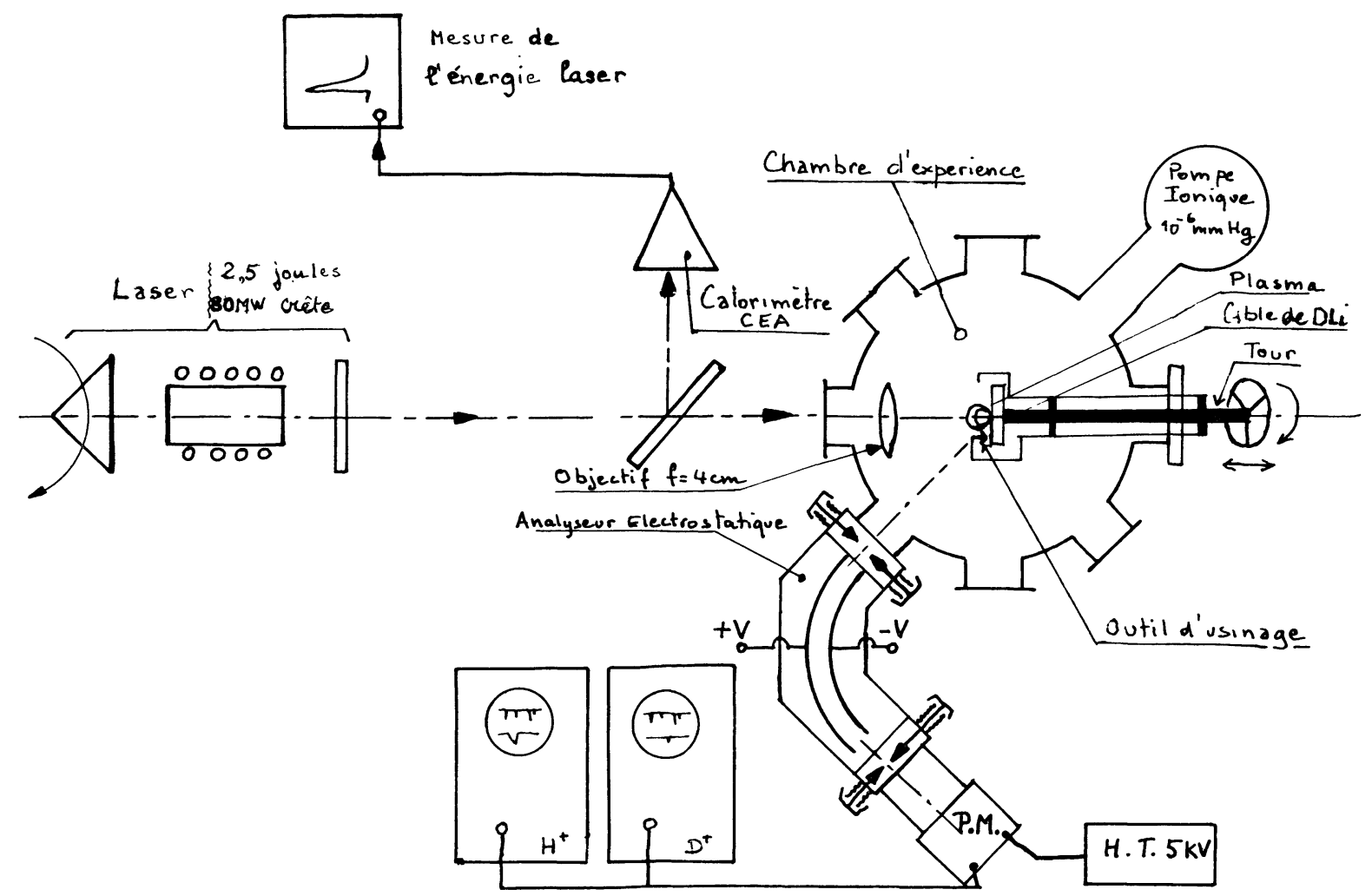

FIG. 1 a.

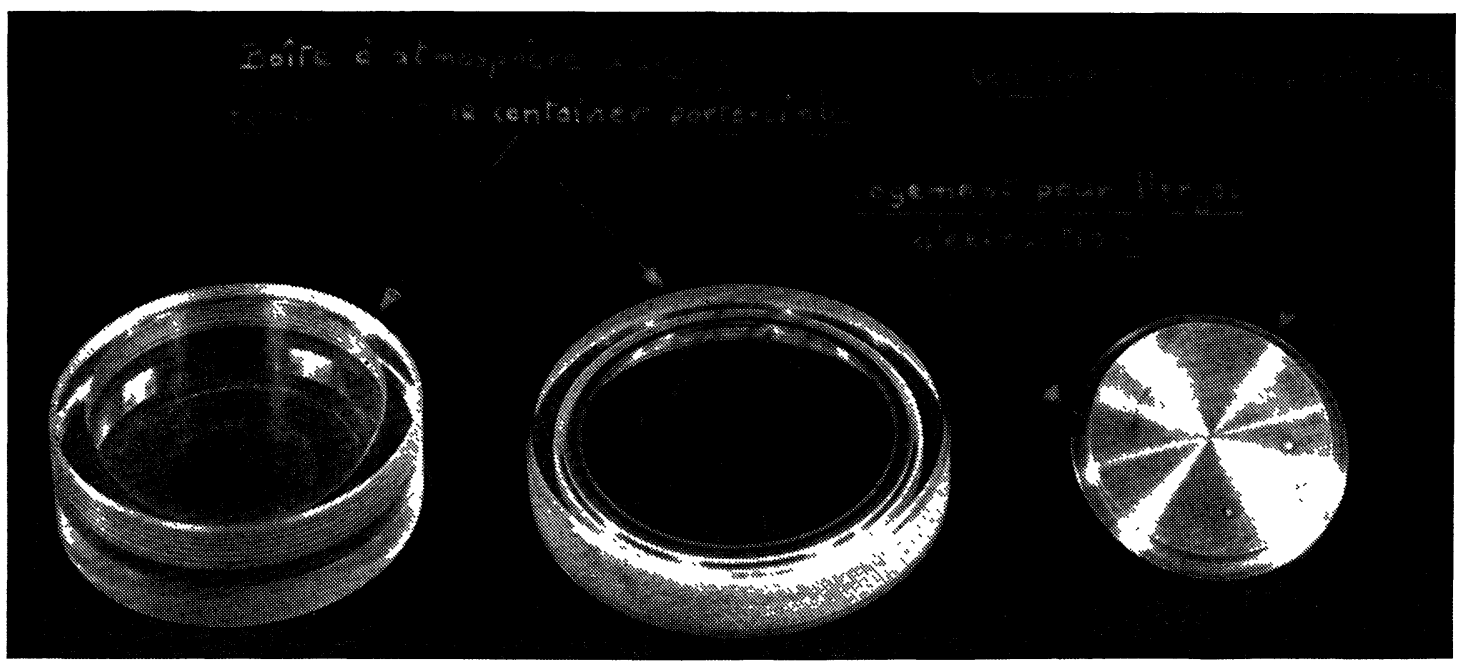

FIG. 1 b. - Containers.

plexiglas à atmosphère d'argon contient un container métallique renfermant la cible en deutérure de lithium également en atmosphère d'argon. b) Usinage DE LA GIBLE sous vide. - Le container métallique renfermant la cible est fixé sur le tour représenté figure $1 \mathrm{c}$. Ce tour, adapté à l'un des 


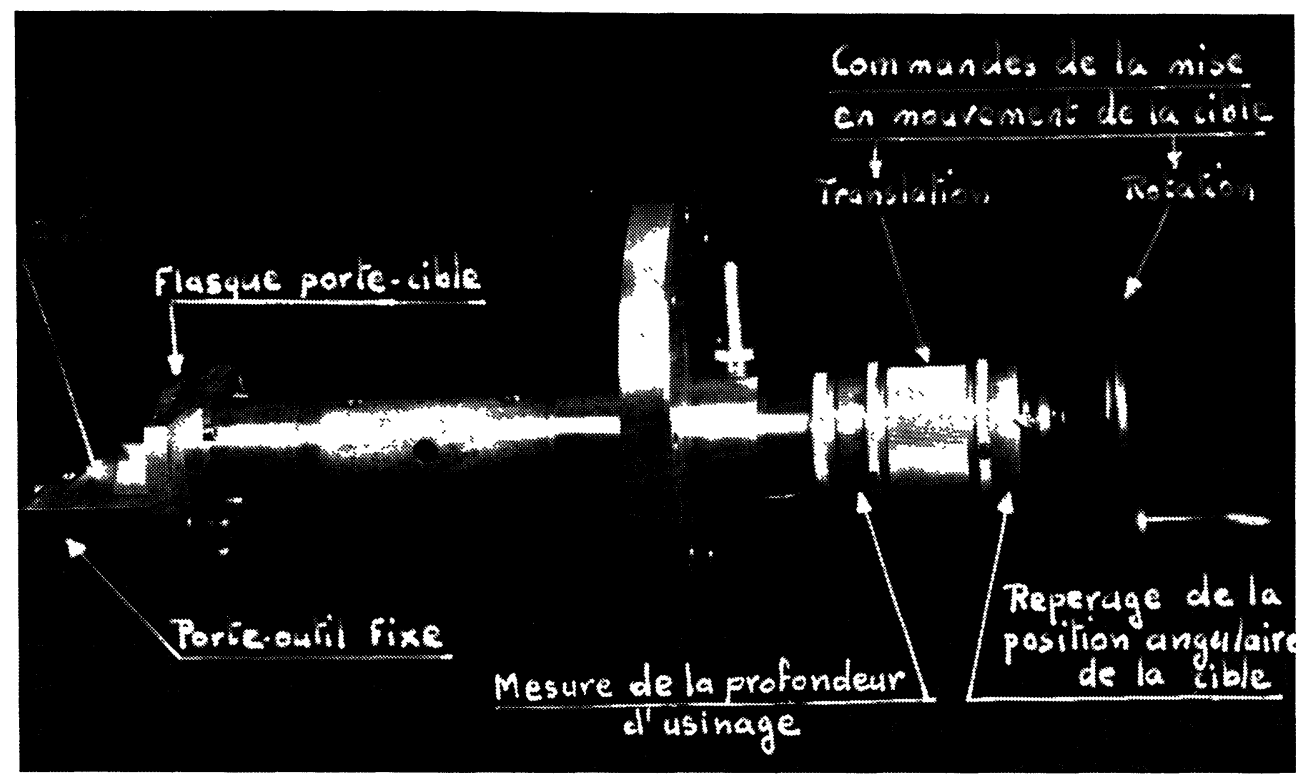

FIG. 1 c. - Vue d'ensemble du porte-cible.

queusots de la chambre d'interaction où l'on maintient une pression résiduelle de $10^{-6}$ torr, permet l'usinage de la surface de la cible au moyen de l'outil représenté figure $1 \mathrm{~d}$. On remarque sur cette photo le dispositif de fixation du container métallique et l'ergot d'extrac-

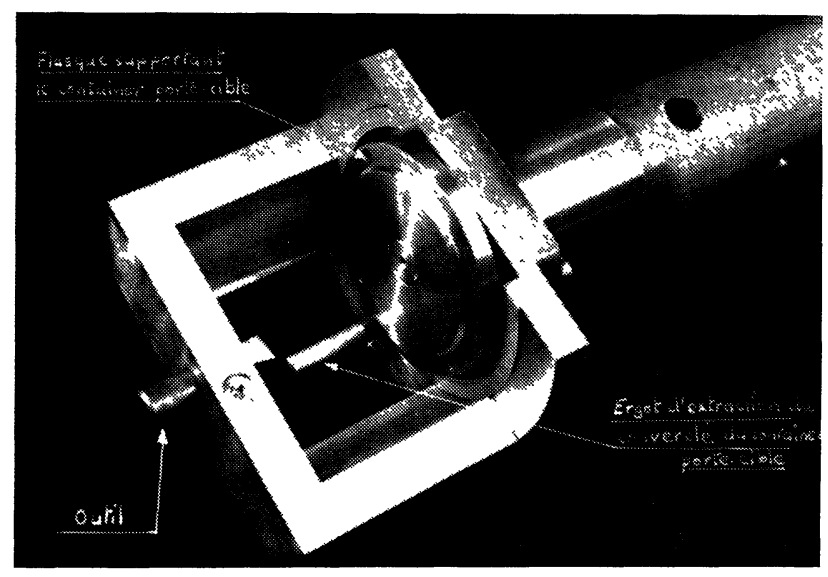

FIG. $1 \mathrm{~d}$. - Détail du porte-outil.

tion qui immobilise le couvercle du container pour le dévisser. On effectue ainsi l'ouverture du container renfermant la cible directement sous vide. Le tour nous permet de prendre des passes de valeur connue au $1 / 100 \mathrm{~mm}$ près à la périphérie de la cible. Le mécanisme de rotation permet de répartir les impacts laser sur la couronne ainsi dégagée.

c) Ensemble laser-objegtif de Gonaentration. Sur le schéma d'ensemble de la figure $1 \mathrm{a}$, nous avons

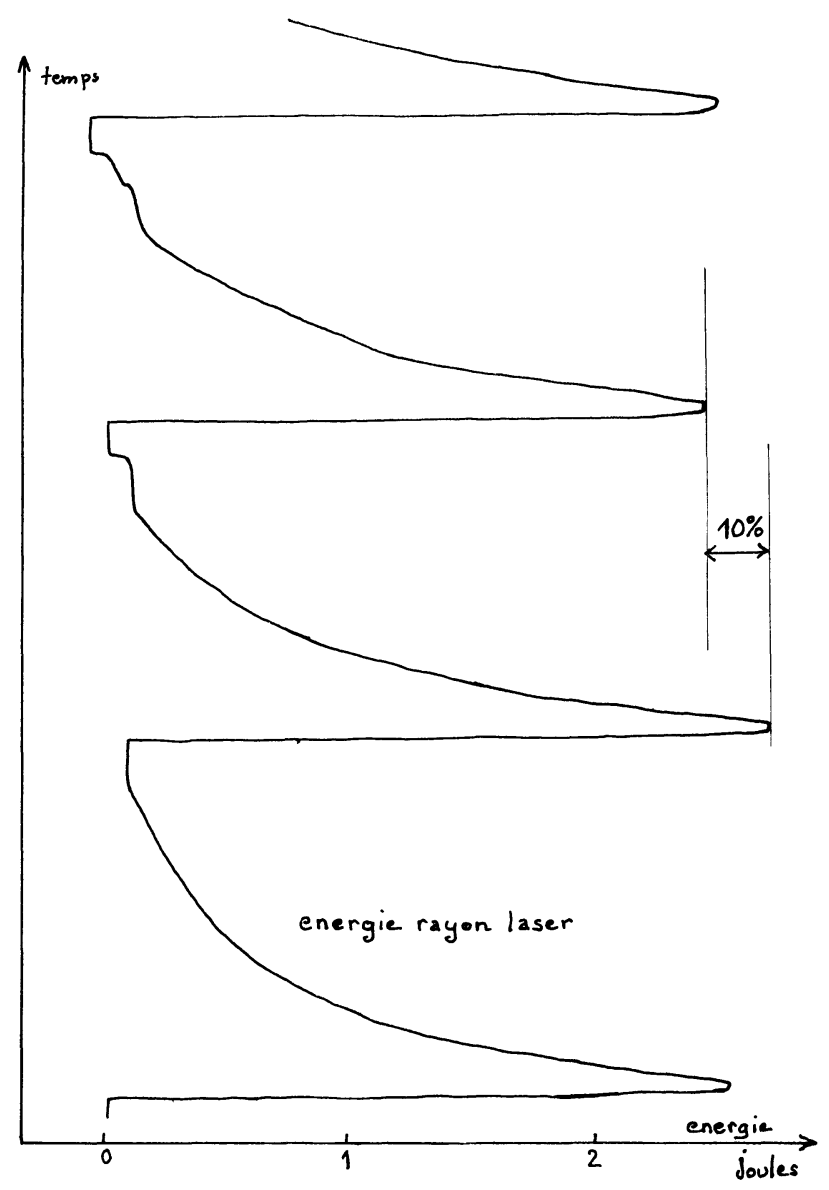

FIG. 1 e. 
schématisé le laser à verre au néodyme utilisé qui se compose d'un oscillateur déclenché par prisme tournant. Ce laser nous délivre un faisceau de diamètre $16 \mathrm{~mm}$, d'énergie 2,5 joules sur la longueur d'onde $\lambda=1,06 \mu \mathrm{m}$. La largeur de l'impulsion à mi-hauteur est de $30 \mathrm{~ns}$, ce qui nous permet d'obtenir une puissance crête de $80 \mathrm{MW}$ environ.

A chaque tir, nous relevons l'énergie laser sur un enregistreur ( fig. $1 \mathrm{e}$ ), en utilisant un calorimètre à cônes de graphite. On peut remarquer (fig. $1 \mathrm{e}$ ) que l'énergie délivrée n'est pas constante mais fluctue d'environ $10 \%$ d'un tir à l'autre.

Le faisceau laser est focalisé sur la cible par l'intermédiaire d'un objectif de $40 \mathrm{~mm}$ de distance focale nous permettant d'obtenir au foyer un éclairement supérieur à $10^{12} \mathrm{~W} / \mathrm{cm}^{2}$.

La figure $1 \mathrm{f}$ représente l'ensemble objectif-cible en deutérure de lithium photographié à travers un des

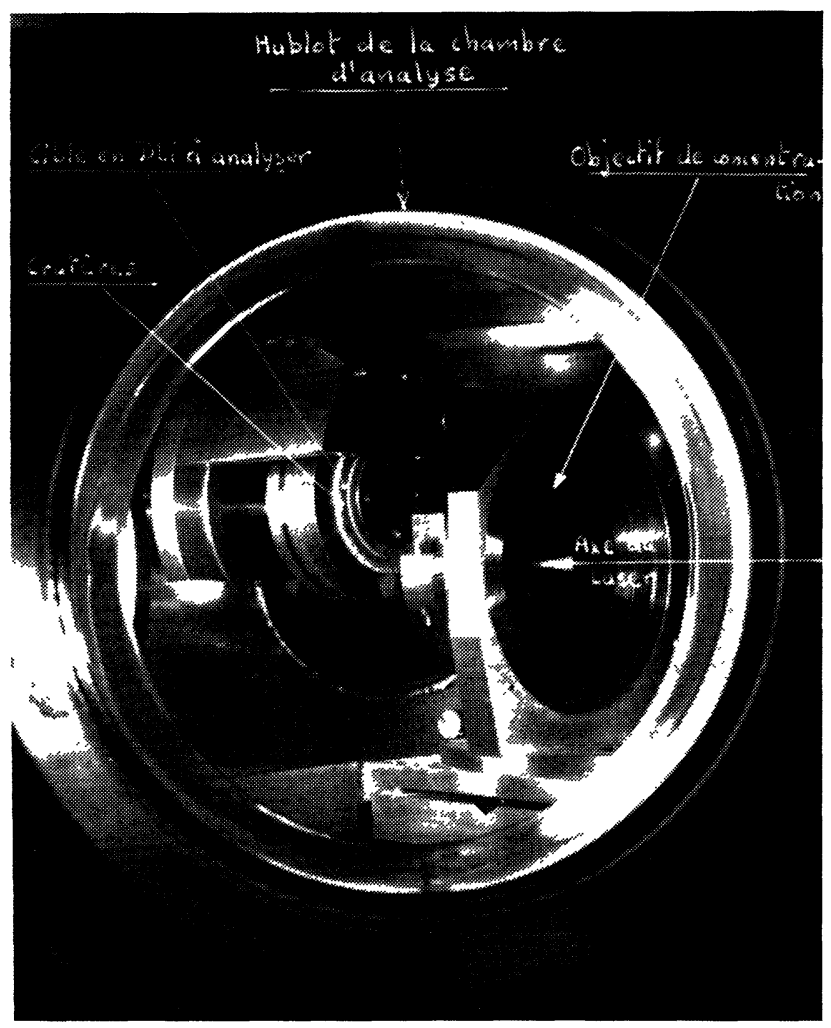

FIG. 1 f. - Ensemble cible-objectif de concentration.

hublots de la chambre d'interaction. On remarquera sur cette photo la cible en DLi usinée sur son pourtour et les cratères creusés par le laser.

2. La détection des ions. - Pour détecter les ions que nous avons créés, nous utilisons un analyseur électrostatique associé à un détecteur d'ions constitué par un multiplicateur d'électrons. a) Description de L'AnAlyseur Élegtrostatique. - Les ions émis par l'impact du faisceau laser ont un certain spectre en énergie [2] et de ce fait un tri en énergie doit être réalisé avant de les détecter. Ce tri s'effectue au moyen de l'analyseur schématisé figure 1 a et dont la figure $1 \mathrm{~g}$ nous montre les électrodes de déviation.

Cet analyseur a déjà été décrit par ailleurs [2], nous ne rappellerons ici que son principe. Les ions d'énergie cinétique $W$ décrivent la trajectoire médiane où le champ électrique a pour valeur :

$E=\frac{V}{d} \quad(d=$ distance entre les deux électrodes $)$.

La force centrifuge $\frac{2 W}{R}$ appliquée $(R=$ rayon moyen de courbure) est égale à la force électrostatique $Z e E\left(Z e=\right.$ charge de l'ion, $\left.e=1,6 \times 10^{-19} \mathrm{Cb}\right)$.

Nous avons donc :

$$
\frac{2 W}{R}=Z e E=Z e \frac{V}{d}
$$

avec $W$ en eV et $V$ en volts.

$$
\frac{W}{Z}=\frac{R}{2 d} \cdot V=k . V
$$

(dans notre cas $k=5$ ).

Tous les ions ayant même rapport $\frac{W}{Z}$ traversent l'analyseur lorsque la tension à ses bornes $V$ vérifie la relation (2).

La séparation en masse s'effectue par temps de vol. En effet, les ions sont émis lors de l'arrivée de l'impulsion laser sur la cible et doivent, avant d'être détectés, parcourir une distance $L$. Si $W=\frac{1}{2} M v^{2}$ est l'énergie cinétique d'un ion de masse $M$ et de vitesse $v$, son temps de vol $\tau$ est donné par :

$$
\tau=\frac{L}{\sqrt{\frac{2 W}{M}}}
$$

d'après (2), nous avons :

$$
\tau=\frac{L}{\sqrt{\frac{2 k Z V}{M}}}=\frac{L}{\sqrt{2 k V}} \sqrt{\frac{M}{Z}}
$$

pour une énergie d'analyse $W$ donnée, $V$ est constant, donc finalement :

$$
\tau=k^{\prime} \sqrt{\frac{M}{Z}} .
$$

Le temps de vol nous permet de séparer les ions selon leur rapport $\frac{M}{Z}$; nous avons réalisé ainsi un spectromètre de masse où nous enregistrons les divers « pics » ioniques sur un oscilloscope. 


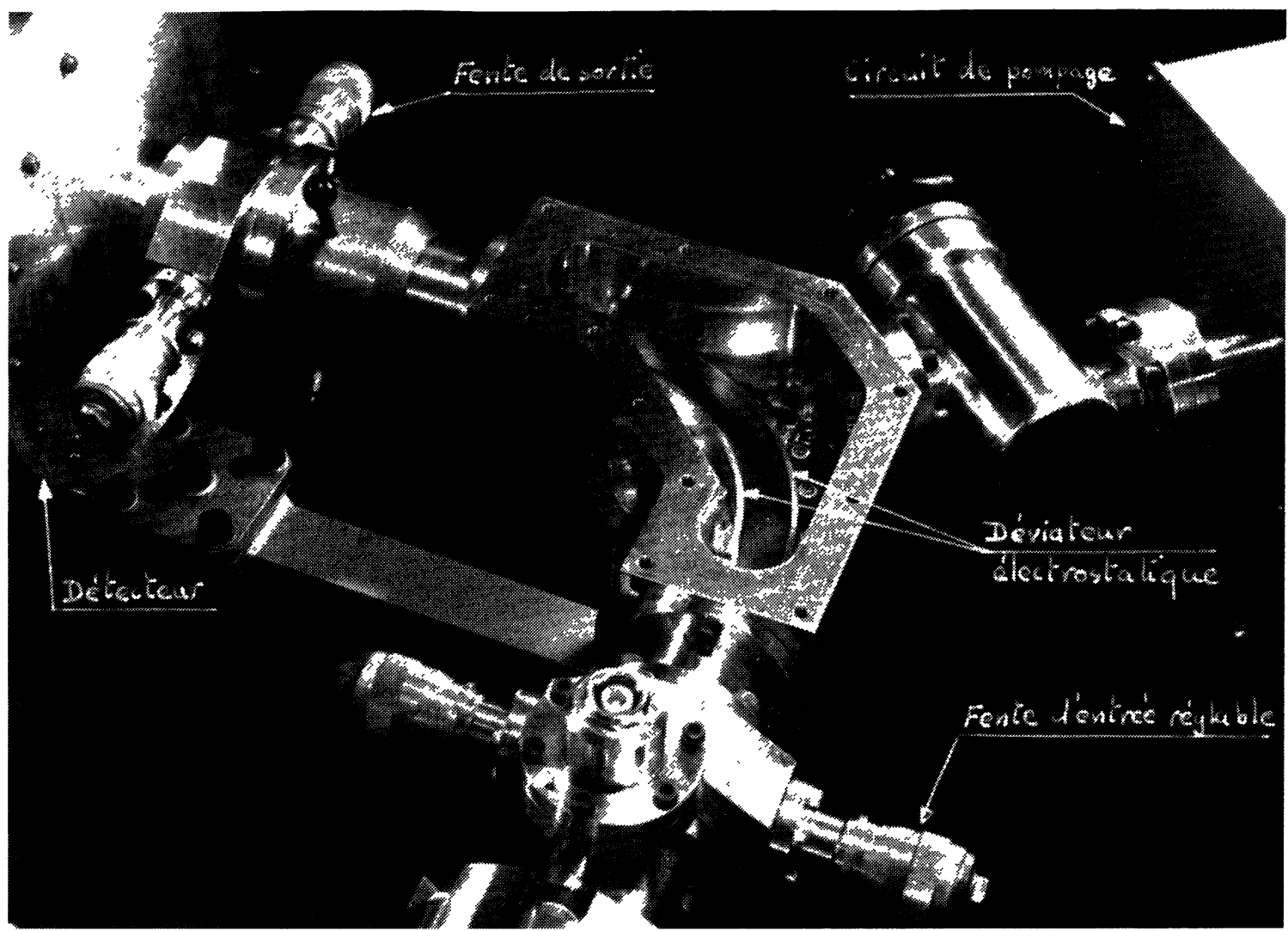

FIg. 1 g. - Déflexion électrostatique de l'analyseur.

b) Résolution du SPectromètre de masse. - Pour calculer la résolution en masse du spectromètre, il faut tenir compte de la durée d'émission des ions au niveau de la cible $\Delta \tau_{\mathrm{s}}$. Dans ces conditions, la largeur de l'impulsion ionique détectée est égale à :

$$
\left[\Delta \tau_{\mathrm{r}}^{2}+\Delta \tau_{\mathrm{s}}^{2}\right]^{1 / 2}=\Delta \tau
$$

avec $\Delta \tau_{\mathrm{r}}$ l'élargissement dû à la résolution en énergie $R_{w}$ de l'analyseur. Nous avons :

$$
R_{w}=\frac{W}{\Delta w}=\frac{1}{2} \frac{v}{\Delta v}=\frac{a_{0}}{2\left(G \times f_{\mathrm{e}}+f_{\mathrm{s}}\right)}
$$

avec $G=$ grandissement de l'analyseur $=1,6$,

$f_{\mathrm{e}}$ et $f_{\mathrm{s}}=$ largeurs respectives des fentes d'entrée et de sortie. Dans notre cas $f_{\mathrm{e}}=0,5 \mathrm{~mm}$ et $f_{\mathrm{s}}=0,8 \mathrm{~mm}$,

$a_{0}=100 \mathrm{~mm}=$ rayon moyen de l'analyseur,

$W=$ énergie cinétique analysée,

$v=$ vitesse des ions analysés.

Si $L$ est la longueur de la base de temps de vol $(L=118,7 \mathrm{~cm})$, nous avons pour un ion de masse $M$ :

$$
\Delta \tau_{\mathrm{r}}=\frac{L \sqrt{M}}{2 \sqrt{2 W}} \times \frac{1}{R_{w}} .
$$

Pour que les impulsions ioniques produites par les ions de masse $M_{1}$ et $M_{2}$ soient séparées, il faut que les temps de vol correspondants $\tau_{1}$ et $\tau_{2}$ soient tels que :

$$
\tau_{2}-\tau_{1} \geqslant \frac{1}{2}\left[\Delta \tau_{\mathrm{r}}^{2}+\Delta \tau_{\mathrm{s}}^{2}\right]^{1 / 2} \quad \text { si } \quad M_{2}>M_{1}
$$

ou :

$$
\begin{aligned}
& \tau_{2}=\frac{L \sqrt{M_{2}}}{\sqrt{2 W}} \\
& \tau_{1}=\frac{L \sqrt{M_{1}}}{\sqrt{2 W}}
\end{aligned}
$$

d'après (4) et (5), nous avons :

$\frac{L}{\sqrt{2 W}}\left[\sqrt{M_{2}}-\sqrt{M_{1}}\right] \geqslant \frac{1}{2}\left[\left(\frac{L \sqrt{M_{1}}}{2 \sqrt{2 W} \cdot R_{w}}\right)^{2}+\Delta \tau_{\mathrm{s}}^{2}\right]^{1 / 2}$.

On pose :

$$
\Delta M=M_{2}-M_{1}
$$

alors :

$$
\begin{aligned}
& \sqrt{M_{2}}-\sqrt{M_{1}}=\frac{M_{2}-M_{1}}{\sqrt{M_{2}}+\sqrt{M_{1}}}=\frac{\Delta M}{2 \sqrt{M_{1}}} \\
& \text { car } \Delta M \ll M_{1} .
\end{aligned}
$$


Nous écrivons (11) en tenant compte de (13) :

$$
\frac{M_{1}}{\Delta M} \leqslant \frac{2 R_{w}}{1+R_{w}^{2} \cdot \Delta \tau_{\mathrm{s}}^{2} \times \frac{2 W}{M_{2} L^{2}}} 1 / 2 .
$$

En tenant compte de (5) dans un cas plus général, on peut donner la résolution en masse $R_{M}$ :

$$
R_{M}=\frac{M}{\Delta M}=\frac{2 R_{w}}{\left[1+R_{w}^{2} \cdot \frac{\Delta \tau_{\mathrm{s}}^{2}}{\tau^{2}}\right]^{1 / 2}}
$$

On constate que la résolution en masse $R_{M}$ est proportionnelle à la résolution en énergie $R_{w}$, mais il s'introduit un terme correctif dû à la durée d'émission au niveau de la source.

Expérimentalement, nous avons trouvé que $\Delta \tau_{\mathrm{s}}$ est de l'ordre de $10^{-7} \mathrm{~s}$. Pour que le terme correctif devienne négligeable, il faut que :

$$
R_{w}^{2} \times \frac{\Delta \tau_{\mathrm{s}}^{2}}{\tau^{2}} \ll 1
$$

on prendra par exemple :

$$
R_{w}^{2} \times \frac{\Delta \tau_{\mathrm{s}}^{2}}{\tau^{2}}=10^{-1}
$$

soit :

$$
\tau^{2}=10 R_{w}^{2} \cdot \Delta \tau_{\mathrm{s}}^{2}
$$

D'après (2) et les valeurs données, on a $R_{w}=30$. Ceci nous impose une valeur limite inférieure du temps de vol de :

$$
\tau=9,5 \times 10^{-6} \mathrm{~s} .
$$

La longueur de la base de temps de vol étant fixée, la limite inférieure au-delà de laquelle l'influence de la source devient négligeable est :

$$
v \leqslant \frac{L}{\tau}=\frac{118,7}{9,5 \times 10^{-6}}=1,25 \times 10^{7} \mathrm{~cm} / \mathrm{s} .
$$

Dans ces conditions :

$$
R_{M} \simeq 2 R_{w}
$$

et pour $R_{w}=30$, nous avons $R_{M} \simeq 60$.

D'après nos conditions d'expérience, nous pouvons séparer les masses 60-61 par exemple, ce qui constitue une performance nettement supérieure à la résolution nécessaire pour séparer $\mathrm{H}^{+}$et $\mathrm{D}^{+}$.

c) Détegtion PAR MUltipliaAteur D'ÉleGtrons. Pour détecter les ions à la sortie de l'analyseur, nous utilisons un multiplicateur d'électrons XP 1331 Radiotechnique. Le gain du multiplicateur est une fonction du coefficient d'émission secondaire $\delta$ du convertisseur.
Ce coefficient $\delta$ varie selon les caractéristiques du faisceau ionique incident, en particulier avec :

- l'énergie cinétique,

- la charge,

- la masse atomique,

- le numéro atomique des ions.

D'après les travaux de F. Hoffert et ses collaborateurs [6], le gain augmente linéairement avec l'énergie pour des ions d'énergie comprise entre 1,2 et $5 \mathrm{keV}$. Dans notre cas, après la fente de sortie de l'analyseur, les ions sont post-accélérés par une haute tension de $4 \mathrm{kV}$. D'autre part, ces ions ont une gamme d'énergie allant de $300 \mathrm{eV}$ à $700 \mathrm{eV}$ environ, donc leur spectre d'énergie lorsqu'ils arriveront sur le convertisseur s'étendra de 4,3 à $4,7 \mathrm{keV}$. Ce spectre étroit sera d'ailleurs le même pour les ions $\mathrm{H}^{+}$et $\mathrm{D}^{+}$. La charge des ions à détecter étant la même ainsi que le numéro atomique, il reste l'influence de la masse. Nous verrons dans le paragraphe suivant que les spectres en énergie relevés pour $\mathrm{H}^{+}$et $\mathrm{D}^{+}$sont tous deux centrés sur la même énergie de $500 \mathrm{eV}$; or, d'après les travaux déjà cités, on constate qu'à énergie égale le gain peut être différent suivant les masses des isotopes. Il s'introduit alors une erreur systématique. Ces résultats contradictoires [6], [7], [8] sur les variations de gain font que seul un étalonnage direct du détecteur à l'aide d'une source à ions peut nous renseigner à ce sujet : cet étalonnage très délicat est prévu ultérieurement.

Nous pouvons penser que le rapport des charges $\frac{\mathrm{H}^{+}}{\mathrm{D}^{+}}$ émises par le plasma et collectées à la sortie du détecteur n'est pas altéré par ce dernier, et on posera $G_{\mathrm{H}^{+}}=G_{\mathrm{D}^{+}}$.

\section{RESULTATS EXPERIMENTAUX}

1. Tracé des spectres en énergie des ions $\mathrm{H}^{+}$et $\mathrm{D}^{+}$. - Nous venons de voir que la séparation en masse des ions se fait par différence de temps de vol. Cet écart temporel des signaux ioniques relatifs aux ions $\mathrm{H}^{+}$et $\mathrm{D}^{+}$apparaît sur l'oscillogramme de la figure 2 .

On remarque :

a) Sur la trace supérieure, l'ensemble des ions détectés par le multiplicateur d'électrons ayant une énergie de $350 \mathrm{eV}$. On vérifie que les instants d'apparition de ces ions comptés depuis le début du balayage déclenché par l'impulsion laser sont proportionnels à $\sqrt{\frac{M}{Z}}$.

On note la présence des isotopes ${ }^{6} \mathrm{Li}$ et ${ }^{7} \mathrm{Li}$ une et deux fois ionisés et d'impuretés (oxygène et carbone).

b) Sur LA TRAGe INFÉRIEURE, nous avons utilisé le balayage étalé, seul apparaît le signal correspondant aux ions deutérium d'énergie $350 \mathrm{eV}$. On constate 


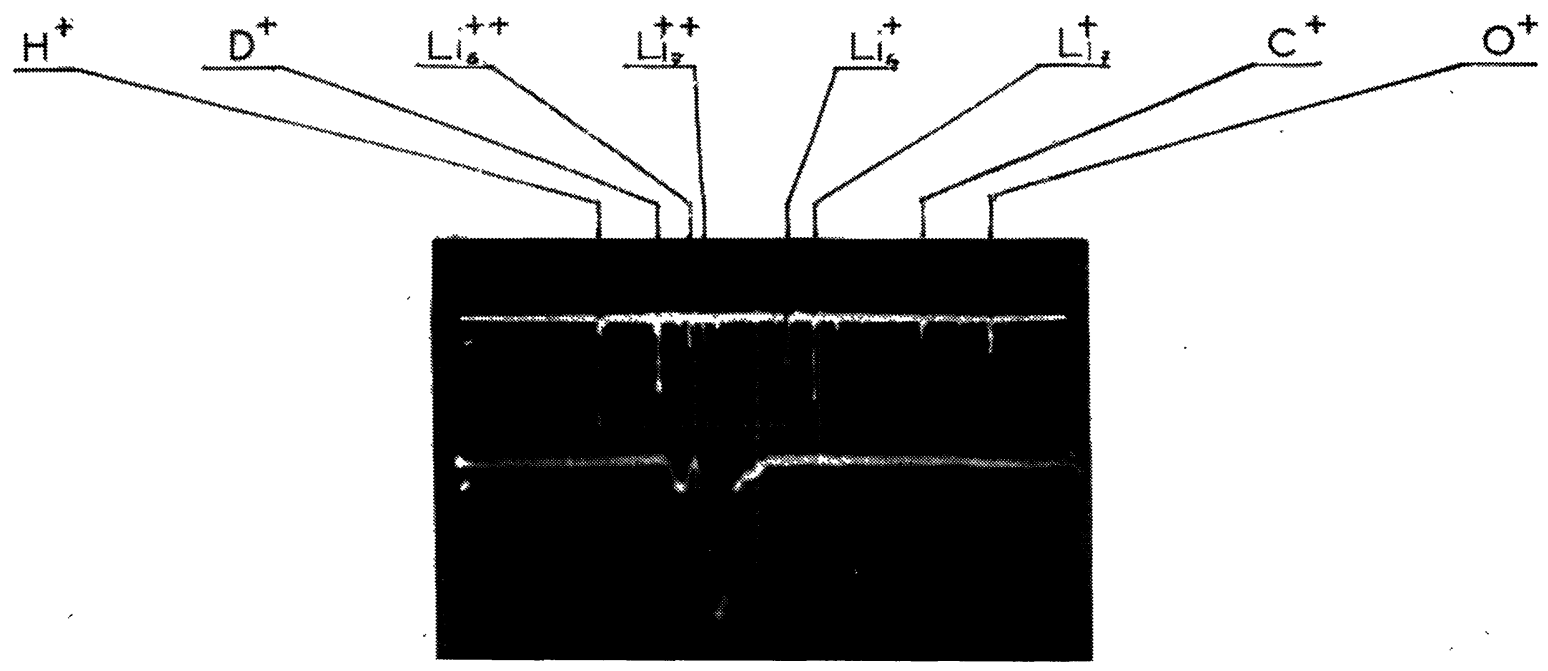

FIG. 2. - Analyse isotopique de DLi :

Trace supérieure : ensemble des ions émis par la cible DLi, $2 \mu \mathrm{s} /$ div., $0,5 \mathrm{~V} / \mathrm{div}$.

Trace inférieure : pic du $\mathrm{D}^{+}, 100 \mathrm{~ns} /$ div., 0,2 V/div.

sur la figure un dédoublement du pic de deutérium. Sans que l'on puisse encore en expliquer clairement les raisons, il semble que ceci soit à rattacher au mécanisme d'émission des ions par le plasma formé lors de l'interaction du faisceau laser avec la cible. En intégrant cette impulsion, nous déterminons la charge collectée égale au produit du gain du multiplicateur par le nombre d'ions deutérium incidents. En faisant varier l'énergie d'analyse, nous avons tracé les spectres de la figure 3 pour les ions $\mathrm{H}^{+}$et $\mathrm{D}^{+}$pour une profondeur donnée.

2. Détermination de la concentration isotopique en fonction de la profondeur. - Par intégration de chacun des spectres de la figure 3 , nous obtenons $\eta$ qui est le rapport du nombre d'ions $\mathrm{H}^{+}$et $\mathrm{D}^{+}$arrivant sur le détecteur, car on considère le gain identique pour les deux types d'ions.

Appliquons la loi de Saha aux deux isotopes.

Dans le plasma, les densités $n$ sont telles que :

donc :

$$
\frac{n_{\text {(ions) }} \cdot n_{\text {electrons }}}{n_{\text {at. neutres }}}=C \mathrm{e}^{-V_{i} / k T}
$$

$$
\frac{n_{\mathrm{H}^{+}} \cdot n_{\mathrm{e}}}{n_{\mathrm{H}}}=C \mathrm{e}^{-V_{i / k T}} \quad \text { et } \quad \frac{n_{\mathrm{D}^{+}} \cdot n_{\mathrm{e}}}{n_{\mathrm{D}}}=C \mathrm{e}^{-\nabla_{i / k T}}
$$

or $V_{i}$, le potentiel d'ionisation, est le même pour $\mathrm{H}$ et $\mathrm{D}$, et $C=\frac{2 \pi m k T^{3 / 2}}{h^{3}}$ ne dépend que de la masse $m$ de l'électron et de la température du plasma, donc :

$$
\eta=\frac{n_{\mathrm{H}^{+}}}{n_{\mathrm{D}^{+}}}=\frac{n_{\mathrm{H}}}{n_{\mathrm{D}}} .
$$

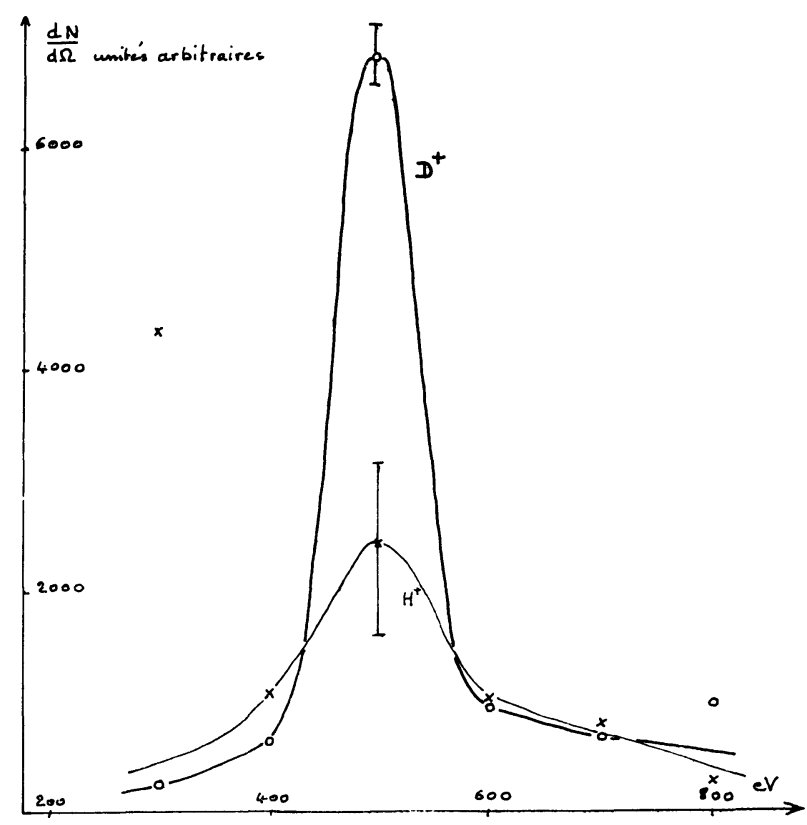

FIG. 3. - Spectres en énergie. Profondeur : $0,1 \mathrm{~mm}$.

Le nombre d'atomes neutres dans le plasma est proportionnel au nombre d'atomes dans le milieu solide, donc $\eta$ est égal au rapport isotopique dans la région de la cible analysée.

Nous avons porté sur la figure 4 la variation de $\eta$ en fonction de la profondeur.

Toutefois, comme les cratères creusés par le laser ont une forme conique d'environ $0,3 \mathrm{~mm}$ de profon- 


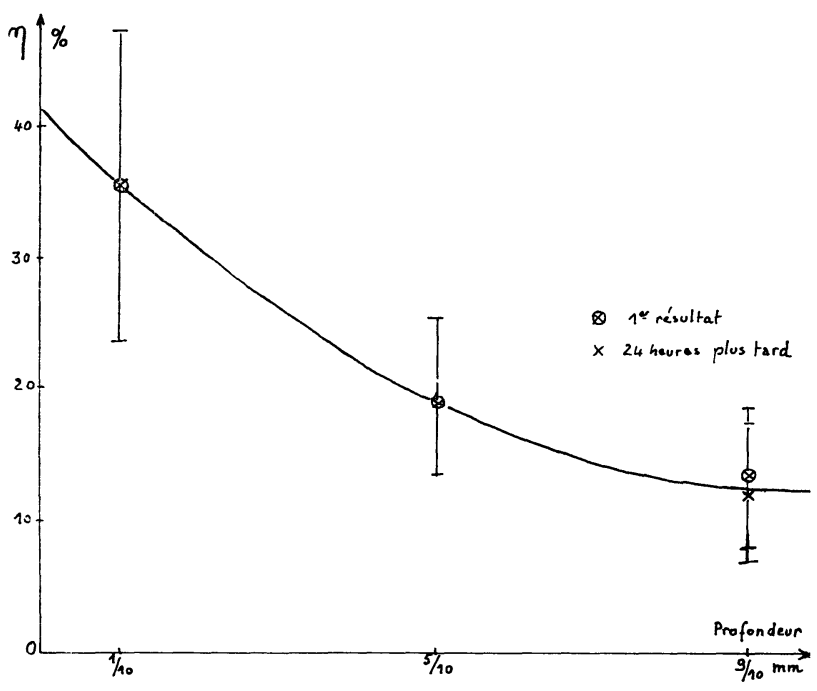

FIG. 4. - Rapport H/D dans DLi.

deur, nous avons pris comme profondeur moyenne d'analyse le barycentre de ce cône qui se trouve alors à $0,1 \mathrm{~mm}$ de la surface de la cible.

Nous remarquerons que la concentration en atomes d'hydrogène est deux fois plus forte en surface qu'à cœur.

3. Précisions des mesures. - a) HydrogénAtion EN GOURS D'ANALYSE. - Les précautions prises afin d'obtenir un vide propre, à savoir la suppression des vapeurs d'huile par l'utilisation d'une pompe primaire à absorption, puis d'une pompe ionique, nous ont permis de vérifier que pendant un intervalle de temps de 24 heures le rapport des concentrations isotopiques ne varie pas en surface de l'échantillon. Ce résultat est signalé sur la figure 4 pour une profondeur d'analyse de $0,9 \mathrm{~mm}$.

b) INGERTITUdES D'ORDRE EXPÉRIMENTAL. - La principale source d'erreurs est due aux fluctuations de l'énergie laser d'un tir à l'autre. Ces fluctuations, comme nous l'avons vu, sont de l'ordre de $10 \%$. compte tenu des caractéristiques de l'émission laser, il semble illusoire d'espérer une amélioration sensible de la répétitivité de l'énergie d'un tir à l'autre.

c) IMPRÉGISIONS DUES AU PRINGIPE MÊME DE MESURE. - Elles sont de deux types :

- L'analyseur est placé à $45^{\circ}$ par rapport à l'axe du faisceau laser ( fig. $1 \mathrm{a}$ ). Or, une étude sur la répartition spatiale de l'émission ionique [9] a montré que celle-ci n'était pas isotrope. L'angle solide d'acceptance de l'analyseur est faible $\left(2 \times 10^{-5}\right.$ stéradian $)$, donc il se peut que le nombre d'ions détectés ne soit pas proportionnel au nombre d'ions émis au niveau de la cible. Toutefois, ceci ne doit pas intervenir lorsque, comme nous le faisons ici, nous cherchons un rapport entre deux concentrations.
On peut remédier à cela en dotant la source d'ions à laser d'une optique électronique focalisant tous les ions émis au niveau de la cible sur la fente d'entrée de l'analyseur [10].

- Pour tracer les spectres en énergie représentés figure 3, il est nécessaire d'effectuer plusieurs tirs, donc d'utiliser une surface assez étendue de la cible. Ceci introduit deux causes d'erreur qui sont d'une part la variation de l'énergie laser d'un tir à l'autre, et d'autre part les hétérogénéités locales. Cet inconvénient peut être éliminé en utilisant un spectromètre de masse à paraboles qui, en un seul tir, donne les spectres de tous les ions présents. Pour certaines applications, ceci est intéressant par le fait que l'on peut faire une analyse ponctuelle (la dimension du cratère est celle de la tache de moindre diffusion de l'objectif qui peut atteindre 20 microns).

d) PRÉcision SUR LA PROFondeur ANAlysée. Dans notre expérience, la profondeur des cratères est d'environ $0,3 \mathrm{~mm}$. Cette profondeur peut être diminuée en réduisant l'énergie du laser. Des résultats expérimentaux sont fournis par Ready [11] à ce sujet. D'autre part, comme nous l'avons vu sur la figure 2, des ions multichargés sont également créés (par exemple pour l'isotope du lithium).

La création de ces ions multichargés peut être favorisée ou annulée en choisissant convenablement l'énergie délivrée par le laser, comme l'a montré une étude réalisée sur une cible en carbone [2].

Ceci confère une grande souplesse d'emploi à la source ionique constituée par la focalisation du faisceau laser sur une cible.

e) Précision globale des mesures. - Compte tenu des remarques précédentes, la figure 4 donne la moyenne des résultats sur trois à cinq tirs par points. Les barres d'erreur représentent $\pm 10 \%$ par rapport aux valeurs indiquées.

\section{CONCLUSION}

L'analyse isotopique par ionisation d'une quantité ponctuelle d'un échantillon à l'aide d'un laser et d'un analyseur électrostatique associé à une base de temps de vol semble une méthode simple dont les premiers résultats sur une cible de DLi sont encourageants.

Gette méthode comporte des avantages sur celles habituellement utilisées comme le bombardement ionique avec émission secondaire d'ions [12] et les décharges dans le vide [13], [14]. Elle permet d'obtenir une analyse quantitative très superficielle (quelque $100 \mu)$ et s'applique quel que soit le matériau même isolant ou réfractaire. Il n'y a pas d'effet de mémoire et le traitement de la cible sous vide élimine tout risque de pollution.

Il est théoriquement possible d'obtenir une analyse 
aussi ponctuelle que l'on désire par le choix de l'optique de focalisation du faisceau laser et de son énergie. Ceci est particulièrement intéressant pour l'analyse d'inclusions.

Nous ne sommes limités actuellement que par la divergence $\mathrm{du}$ faisceau laser (tache de $50 \mu$ de diamètre).

L'analyse peut s'appliquer à n'importe quel isotope. Seule la résolution du spectromètre pourra imposer une limite pour les corps de masse élevée.

\section{BIBLIOGRAPHIE}

[1] Ducauze (A.), Tonon (G.) et Veyrié (P.), Étude de l'énergie des ions émis par une cible métallique frappée par le faisceau d'un laser, C. R. Acad. Sci., Paris, 15 novembre 1965, 261, 4039-4041.

[2] Tonon (G.), Spectre de l'énergie des ions émis par Be, C, Mo sous l'action du faisceau d'un laser, C. R. Acad. Sci., Paris, 7 mars 1966, 262, 706-709.

[3] Langer (P.), Tonon (G.), Floux (F.) et DUCAUZE (A.), Laser induced emission of electrons, ions and $\mathrm{X}$ rays from solid targets, I.E.E.E., Journal of Quantum Electronics, September 1966, $Q E$ 2, no 9, 499-506.

[4] Langer (P.), Tonon (G.), Durand (Y.) et Buges (J. C.), Étude chronologique du plasma de béryllium créé par le faisceau d'un laser, Rapport C.E.A., R 3296, 1967.

[5] Nelison (P.), Plasmas créés par un laser, J. Physique, août-septembre 1965, 26, nos $8-9,476$.

[6] Hoffert (F.), Paulus (J.) et AdlofF (J. P.), Influence de la nature et de l'énergie d'un faisceau ionique sur le gain d'un multiplicateur d'électrons utilisé en spectrométrie de masse, Revue Phys. Appl., mars 1966, 1, 43-47.

[7] BARNETTT (C.), The characteristics of an electron multiplier as a detector of heavy particles, Rapport américain, O.R.N.L., 1669, February 15, 1954.
[8] BRANCHER (J. C.), Le spectromètre de masse comme diagnostic de plasma, Thèse présentée à la Faculté des Sciences, Université de Paris, juin 1960.

[9] Ducauze (A.) et Langer (P.), Répartition des particules émises par focalisation d'un faisceau laser sur une cible solide, C. R. Acad. Sci., Paris, 25 mai 1966, série B, 262, 1398-1401.

[10] Dumas (J. L.), Source d'ions pour spectromètre de masse à laser CEN.G/DPC/SIS/SSM nos 66-82, JLD/JG, juin 1966 .

[11] READY (J. F.), Effects due to absorption of laser radiation, $J$. Applied Physics, February 1965, 36, no 2, 462-468.

[12] Castaing (R.) et Slodzian (G.), Microanalyse par émission ionique secondaire (Institut du Pétrole, ed. London, Advances in mass spectrometry, 1966, 3, 91-98).

[13] Halliday (J. S.), Swift (P.) et WolstenHOLME (W. A.), Quantitative analysis by spark source mass spectrometry (Institut du Pétrole, ed. London, Advance in mass spectrometry, 1966, 3, 143-161).

[14] Desjardins (M.) et al., Essais d'analyses absolues par spectrométrie de masse à étincelle (Institut du Pétrole, ed. London, Advances in mass spectrometry, 1966, 3, 131-142). 